\title{
Acoustic resonator based on periodically poled transducers: Concept and analysis
}

\author{
F. Bassignot, ${ }^{1, a)}$ E. Courjon, ${ }^{1}$ G. Ulliac, ${ }^{1}$ S. Ballandras,,${ }^{1, b)}$ J.-M. Lesage,${ }^{2}$ and R. Petit $^{2}$ \\ ${ }^{1}$ FEMTO-ST, UMR 6174, CNRS-UFC-ENSMM-UTBM, Besançon, France \\ ${ }^{2} \mathrm{MC} / \mathrm{CO}, \mathrm{DGA}$, Rennes, France
}

(Received 14 November 2011; accepted 20 February 2012; published online 26 March 2012)

\begin{abstract}
The demand for highly coupled high quality acoustic wave devices for radio-frequency (RF) signal processing based on passive devices has generated a strong innovative activity, yielding the investigation of new excitation principles and waveguide structures. Periodically poled transducers (PPTs) have been recently investigated [E. Courjon et al., J. Appl. Phys. 102, 114107 (2007)], as an alternative to classical interdigital transducers (IDTs) for the excitation and detection of guided acoustic waves. PPTs have two principal advantages compared to IDTs: the robustness of the excitation versus defects or surface contamination and the possibility to excite waves exhibiting a wavelength equal to the poling period. Here a new acoustic resonator concept is suggested, allowing high frequency operation with a simplified package. The idea consists of using a waveguide based on a PPT fabricated on a ferroelectric single-crystal substrate such as lithium niobate or tantalate inserted between two single-crystal substrates allowing the guidance of elastic waves without losses. The concept analysis points out optimum configurations of the structure allowing the excitation of elastic waves compatible with RF applications. (C) 2012 American Institute of Physics.
\end{abstract}

[http://dx.doi.org/10.1063/1.3697596]

\section{INTRODUCTION}

Clocks nowadays are more and more precise and stable and their applications are various: telecommunications, metrology, radars, etc. The most common clock used is a quartz crystal. Quartz devices are manufactured for a frequency range from a few tens of kilohertz to tens of megahertz. For higher frequencies, surface or bulk acoustic wave resonators are well-suited. They are widely used for the radio-frequency signal processing particularly for the stabilization of electric oscillators in the frequency range $50 \mathrm{MHz}$ to $6 \mathrm{GHz}$. Nevertheless, these components are limited by their sensitivity to the environment (in particular by the temperature which is the first cause of oscillator instabilities) and by the encapsulation quality requirements (free surfaces, encapsulation under vacuum). More and more applications such as earth geolocation, navigation, and radar systems need very high-frequency oscillators, spectral purity, and short-term stability. This paper shows an innovative solution to answer the needs in terms of spectral purity, immunity to parasites, packaging simplification, and fabrication robustness. ${ }^{2}$ The suggested structure is based on a periodically poled transducer $(\mathrm{PPT})^{1}$ inserted between two guiding substrates in order to fabricate a waveguide. In this paper, the PPT-based waveguide concept is presented, as well as simulation results obtained using our finite element and boundary element simulation tools. ${ }^{3}$

\section{WAVEGUIDE CONCEPT}

As explained in Sec. I, the structure is based on a PPT implemented on a ferroelectric layer bonded between two

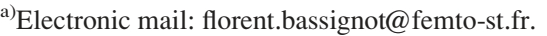

b)Electronic mail: sylvain.ballandras@femto-st.fr.
}

guiding substrates as illustrated in Fig. 1. PPTs have been recently investigated as an alternative to standard interdigital transducers (IDTs) for the excitation and detection of guided acoustic waves. ${ }^{1}$ A PPT corresponds to a periodic alternation of opposite polarization domains. The main advantages of PPTs compared to standard IDTs are the robustness of the excitation versus defects or surface contamination, the opportunity to excite fundamental waves exhibiting an operating frequency twice as high for a same spatial period, and the possibility to excite waves of various polarizations.

In the waveguide concept proposed, elastic waves are excited by a PPT and are trapped within the buried guiding structure. These waves decrease exponentially and propagate without any acoustic losses in the periodic structure. This description is close to the one of interface waves ${ }^{4}$ and fairly coincides with the behavior of an isolated wave. ${ }^{5}$

The transducer consists of two electrically conductive media deposited on each side of a periodically poled ferroelectric layer in order to obtain a capacitive dipole in which elastic waves can be excited by phase construction. These electrodes are also used to bond the transducer on guiding materials. In fact, the wafer bonding technique involves a metallic layer, in our case a gold layer, avoiding the generation of acoustic losses. ${ }^{6}$ Among the possible materials for the transduction, Z-cut lithium niobate $\left(\mathrm{LiNbO}_{3}\right)^{1}$ and $\mathrm{Z}$-cut lithium tantalate $\left(\mathrm{LiTaO}_{3}\right)$ wafers have been studied because both are unipolar materials whose spontaneous polarization lies along the $\mathrm{Z}$ axis. Different guiding materials can guide the elastic waves, in particular silicon, allowing to also integrate the final device with low-cost packaging in microelectronic systems. The scope statement of the new acoustic resonator is summarized by a functional block diagram presented in Fig. 2. 


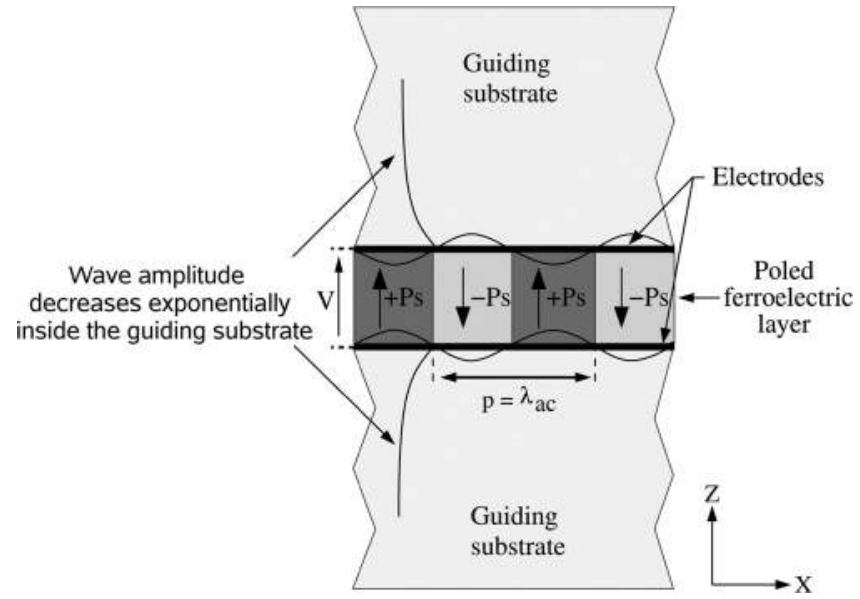

FIG. 1. Scheme of the waveguide concept based on a periodically poled transducer.

\section{ANALYSIS}

In order to validate the principle, to optimize the geometry, and to identify the appropriate materials of the new PPTbased resonator, we present a theoretical analysis of the device including thermal sensitivity computations obtained using our finite element and boundary element simulation tools. ${ }^{3}$ First of all, the analysis of the elastic waves excited by a periodically poled transducer on $\mathrm{LiNbO}_{3}$ and $\mathrm{LiTaO}_{3}$ materials is presented. Then the simulations of PPT-based waveguides are developed.

\section{A. PPT simulations}

A periodically poled transducer is defined by a period (labeled $p$ ) equal to its acoustic wavelength, a thickness $(e)$, an acoustic aperture $(l)$, and a length $(L)$ as presented in Fig. 3. The electrodes exciting the ferroelectric material are not presented on the drawing. They are deposited on the whole surface of the upper and lower faces of the material (in order to create an electrical potential difference).

A two-dimensional structure analysis is employed by assuming an infinite acoustic aperture. As the structure is symmetric and periodic, we can consider a mesh of only one

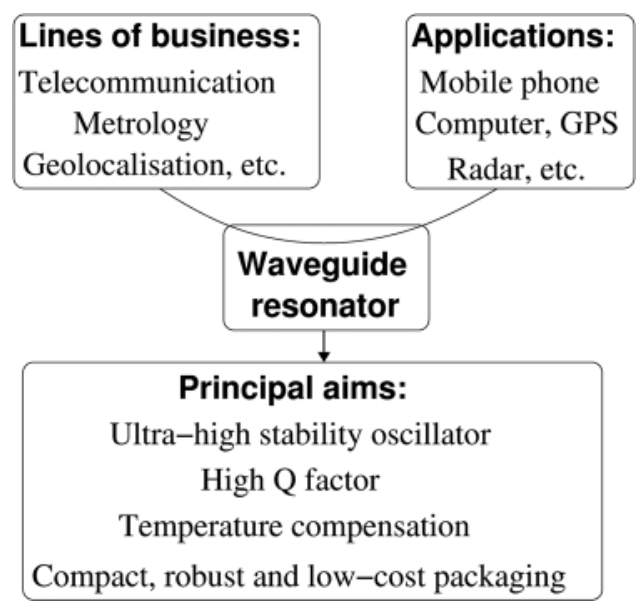

FIG. 2. Functional block diagram of the new acoustic resonator.

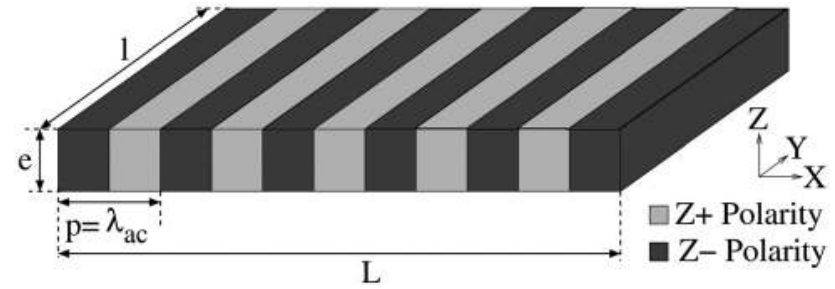

FIG. 3. Scheme of a periodically poled transducer with a definition of its geometrical characteristics.

period of the transducer consisting of two rectangles representing the two opposite ferroelectric domains. The mesh actually used in our simulations is shown in Fig. 4.

Although this mesh is defined in the sagittal $(\mathrm{x}, \mathrm{z})$ plane, shear displacement along $(\mathrm{y}-\mathrm{z})$ are actually taken into account. This area has to be meshed because it presents heterogeneous piezoelectric properties. A $Z+$ polarized domain corresponds to the $(\mathrm{YXl}) / 90^{\circ}$ cut while the second domain (Z-polarized) is a $(\mathrm{YXI}) /-90^{\circ}$ cut. The numbers on the mesh edges correspond to the references characterizing the boundary conditions applied to the mesh limits. In our case, the references labeled " 1 " and " 2 " correspond to the applied electric potential and the references labeled " 3 " and " 4 " are representative of the periodicity conditions. ${ }^{3}$

Once the PPT mesh is defined, one is able to simulate different PPTs on lithium niobate and tantalate. The simulations based on a finite elements analysis allow one to get the harmonic admittance of devices. One can then determine the nature, synchronism frequency, electromechanical coupling, and thermal sensitivity of the excited waves. Figures 5(a) and 5(b) show the harmonic admittances of $500 \mu \mathrm{m}$-thick $\mathrm{LiNbO}_{3}$ and $\mathrm{LiTaO}_{3}$ PPTs, respectively, for an arbitrarily chosen $50 \mu \mathrm{m}$ period. Harmonic admittances are obtained in adding acoustic losses. The order of magnitude of the conductance depends of these losses.

Two main contributions in both configurations are pointed out. For the $\mathrm{LiNbO}_{3}$ PPT, the first mode is excited at $76 \mathrm{MHz}$ (corresponding to a phase velocity $\left(V_{\varphi}\right)$ equal to $3800 \mathrm{~m} \mathrm{~s}^{-1}$ ) and the second one at $131 \mathrm{MHz}\left(V_{\varphi}=6550 \mathrm{~m} \mathrm{~s}^{-1}\right)$. For the $\mathrm{LiTaO}_{3}$ PPT, these contributions are excited to lower frequencies: the first one at $65 \mathrm{MHz}\left(V_{\varphi}=3250 \mathrm{~m} \mathrm{~s}^{-1}\right)$ and the second

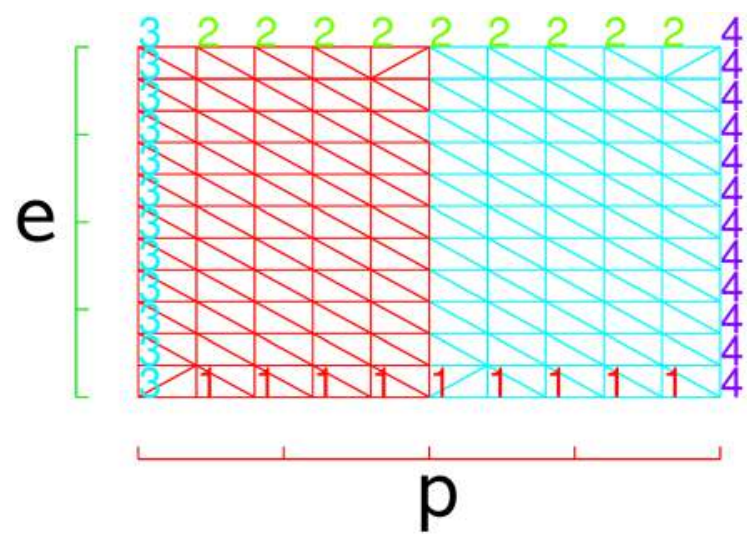

FIG. 4. 2D mesh of a PPT used with our boundary and finite elements simulation tool. 
(a)

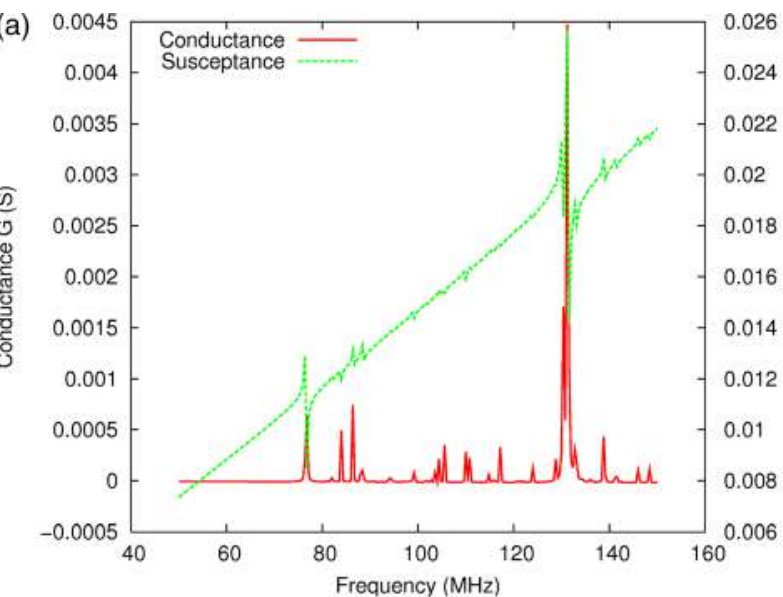

(b)

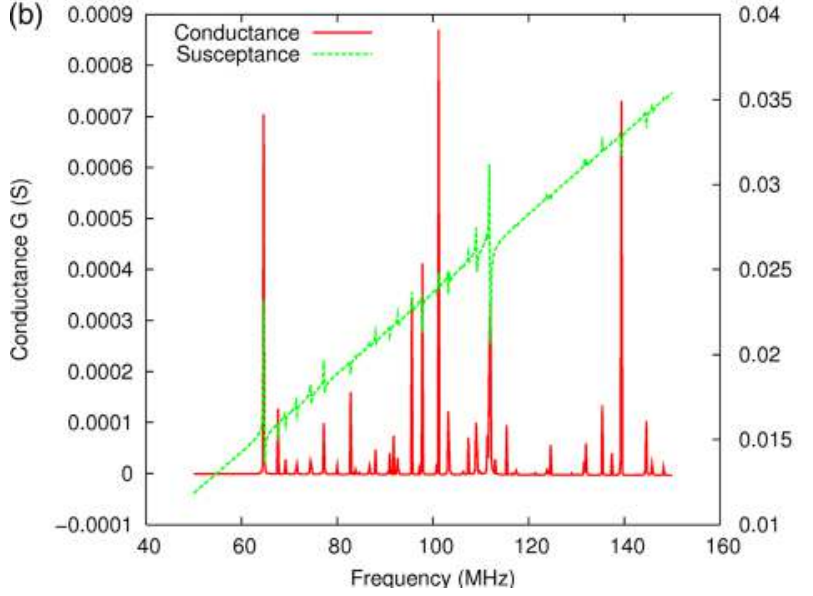

FIG. 5. Harmonic admittances of a $500 \mu$ m-thick $\mathrm{LiNbO}_{3} \mathrm{PPT}$ (a) and for a $500 \mu \mathrm{m}$-thick $\mathrm{LiTaO}_{3}$ PPT (b) for a $50 \mu \mathrm{m}$-period.

one at $112 \mathrm{MHz}\left(V_{\varphi}=5600 \mathrm{~m} \mathrm{~s}^{-1}\right)$. Moreover, one can note the presence of many other modes. In this state, these transducers are then not adapted for the realization of a high spectral purity resonator. The polarization of these modes has been determined by our simulation tool. ${ }^{1}$ The first mode can be compared to Lamb waves. Lamb waves are generated according to the propagation direction by multiple longitudinal and transverse wave reflections on the upper and lower faces of the plate. That wave is then called "elliptical wave." The second mode is close to a longitudinal mode with an extension and compression along the propagation direction.

We have calculated the electromechanical coupling $\left(k^{2}\right)$ and the temperature drift $(T C F)$ for both modes of PPTs $\left(\mathrm{LiNbO}_{3}\right.$ and $\left.\mathrm{LiTaO}_{3}\right)$. Table I summarizes the principal characteristics of these waves.

One can note that the electromechanical coupling coefficients of waves excited by a PPT are equivalent to the ones

TABLE I. Summary of principal characteristics of elliptical and longitudinal waves excited by $\mathrm{LiNbO}_{3}$ and $\mathrm{LiTaO}_{3}$ PPTs.

\begin{tabular}{lcccc}
\hline \hline & Mode polarization & Celerity $\left(\mathrm{m} \mathrm{s}^{-1}\right)$ & $\mathrm{k}^{2}(\%)$ & $\mathrm{TCF}(\mathrm{ppm} / \mathrm{K})$ \\
\hline $\mathrm{LiNbO}_{3} \mathrm{PPT}$ & Elliptical & 3800 & 0.1 & -76 \\
& Longitudinal & 6550 & 0.1 & -81 \\
$\mathrm{LiTaO}_{3} \mathrm{PPT}$ & Elliptical & 3250 & 0.05 & -74 \\
& Longitudinal & 5600 & 0.05 & -49 \\
\hline \hline
\end{tabular}

of Rayleigh waves on quartz (about $0.1 \%){ }^{7}$ Moreover, we point out that those waves are sensitive to the temperature with a TCF lower than $-50 \mathrm{ppm} / \mathrm{K}$ in the case of $\mathrm{Z}$-cut $\mathrm{LiTaO}_{3}$. In order to fabricate a resonator, we must of course reduce this temperature drift and try to increase the electromechanical coupling. For that purpose, we insert the PPT between two guiding substrates. Some simulations on this concept are presented in Sec. III B.

\section{B. PPT-based waveguide simulations}

Figure 6 shows a scheme of only one period of a PPTbased waveguide. The previously studied PPT is now bonded on homogeneous semi-infinite guiding materials on each side. In this study, we particularly consider silicon as a guiding material because silicon is low-cost and well-suited in microelectronic systems. The chosen crystallographic orientation of silicon is (100) because the transverse waves, called surfaceskiming bulk waves (SSBWs), are well-suited in this cut, allowing the guidance of waves excited by the transducer.

The modeling of this structure has to take into account the heterogeneous part of the problem (corresponding to the PPT) and the homogeneous part as well (guiding materials). We thus use a mesh to define the PPT by means of finite elements but also boundary elements based on Green functions to model the guiding substrates. ${ }^{3}$ As explained in Sec. II, a gold layer is used to bond wafers and also as an electrode in order to excite the ferroelectric material. Its thickness has been fixed at $250 \mathrm{~nm}$. The same boundary conditions as for the structure of Fig. 4 have been applied on the PPT mesh. Radiation conditions on references labeled 1 and 2 are added in order to simulate the guiding materials.

Figure 7 shows the harmonic admittances obtained for various thicknesses of $\mathrm{LiNbO}_{3}$ PPT (period $=50 \mu \mathrm{m}$ ) bonded between two silicon substrates.

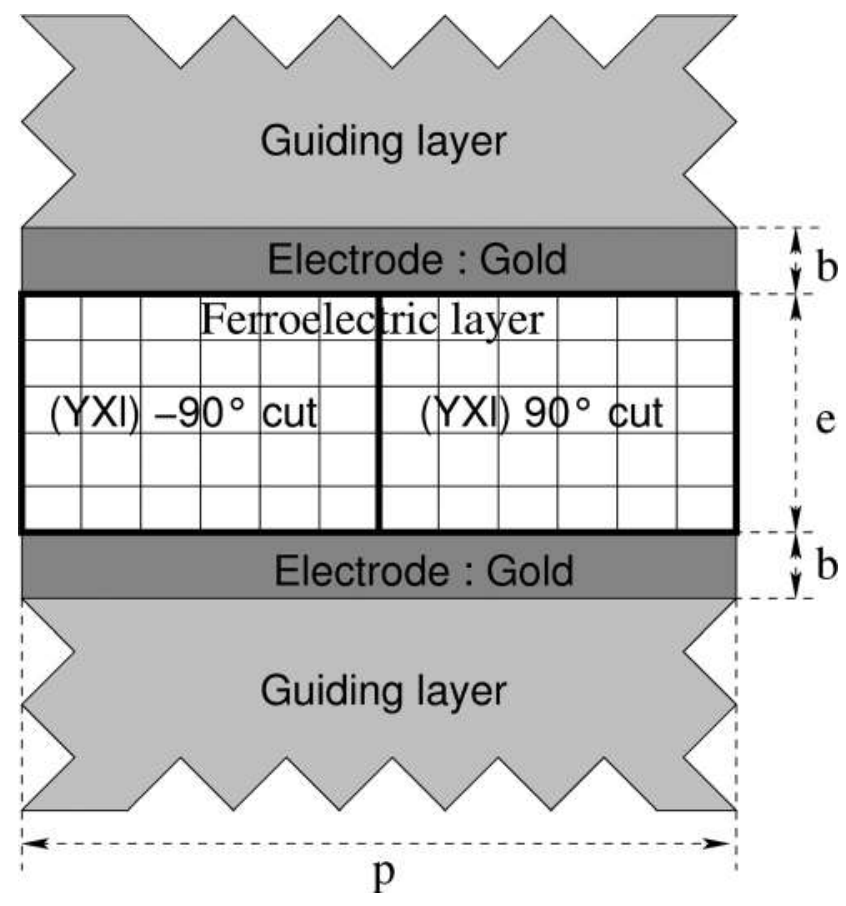

FIG. 6. Scheme of a PPT-based waveguide. 


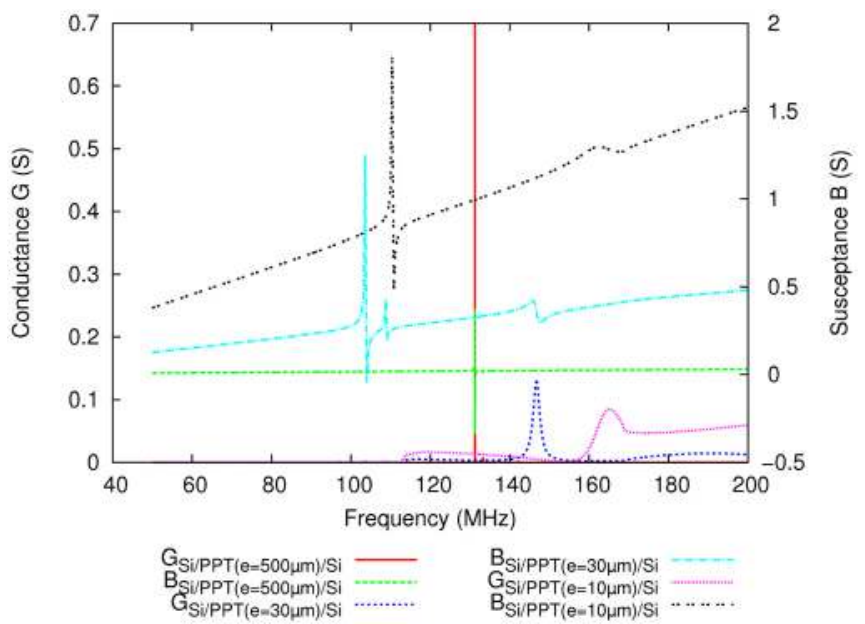

FIG. 7. Harmonic admittances of a Si/LiNbO 3 PPT $(\mathrm{p}=50 \mu \mathrm{m}) / \mathrm{Si}$ waveguide for different PPT thicknesses $(\mathrm{e}=500,30$, and $10 \mu \mathrm{m})$.

In comparison with the harmonic admittance of a 500 $\mu$ m-thick $\mathrm{LiNbO}_{3}$ PPT and for a same PPT thickness, only one of the two previously emphasized modes is excited. This mode corresponds to the longitudinal one with a phase velocity equal to $6550 \mathrm{~m} \mathrm{~s}^{-1}$ and a $0.15 \%$ electromechanical coupling. The elliptical wave cannot propagate in such a waveguide structure because the guiding materials constrain the surface of the PPT. When the PPT thickness is reduced, the longitudinal wave radiates in the guiding materials and another wave is guided at a lower frequency. We can note that this wave is isolated for a $10 \mu \mathrm{m}$ thick PPT, with a pure spectral signal. That is why this wave has been called isolated wave. Figure 8 shows the analysis of dispersion properties of this isolated wave for a $50 \mu \mathrm{m}$-period PPT.

For a $500 \mu \mathrm{m}$-thick PPT, the isolated wave is excited but its electromechanical coupling is about zero. In this configuration, the polarization of this wave is mainly elliptical, explaining the very small value of electromechanical coupling. When the PPT thickness decreases, the electromechanical coupling increases and the wave becomes mainly longitudinal. We can see an optimum point with a maximum

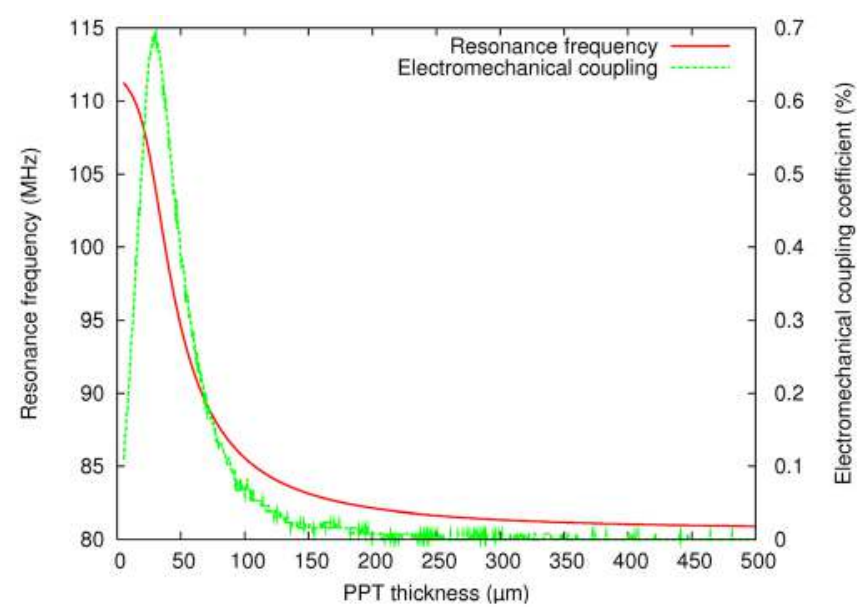

FIG. 8. Evolution of the isolated mode synchronism frequency and electromechanical coupling for PPT thicknesses ranging from 1 to $500 \mu \mathrm{m}$ for a $50 \mu$ m-period PPT. electromechanical coupling $\left(\mathrm{k}^{2}=0.7 \%\right)$ for a $30 \mu \mathrm{m}$-thick $\mathrm{LiNbO}_{3}$ PPT. Once the mode is identified, the evolution of its electromechanical coupling coefficient can be tracked along thickness (e)/period (p) ratios ranging from 0.1 to 2 for several periods. Figure 9 (a) presents the results for a $\mathrm{Si} /$ $\mathrm{LiNbO}_{3} \mathrm{PPT} / \mathrm{Si}$ waveguide and Fig. 9(b) presents the results for a $\mathrm{Si} / \mathrm{LiTaO}_{3} \mathrm{PPT} / \mathrm{Si}$ configuration.

Both structures exhibit a maximum electromechanical coupling for a $0.6(\mathrm{e} / \mathrm{p})$ ratio. Nevertheless, the maximum of the electromechanical coupling of a $\mathrm{Si} / \mathrm{LiTaO}_{3} \mathrm{PPT} / \mathrm{Si}$ structure is quasi-stable at about $1 \%$ for any of the PPT period, whereas for a $\mathrm{Si} / \mathrm{LiNbO}_{3} \mathrm{PPT} / \mathrm{Si}$ structure, the electromechanical coupling falls when the period decreases. Consequently, a $\mathrm{LiTaO}_{3}$ PPT seems a better choice to fabricate a high frequency resonator. However, we can also use a $\mathrm{LiNbO}_{3}$ PPT for a transducer period higher than $30 \mu \mathrm{m}$.

The temperature induced frequency drift of waves excited by a waveguide has also been studied. Figure 10(a) presents the TCF evolution for the longitudinal wave, whereas Fig. 10(b) shows the one for the isolated wave, both for a $\mathrm{Si} / \mathrm{LiNbO}_{3}$ PPT $(\mathrm{p}=50 \mu \mathrm{m}) / \mathrm{Si}$ structure for different PPT thicknesses.

The thickness values of these figures are just a trend because our calculations do not consider the thermodifferential constraints effects. One can note that the TCF
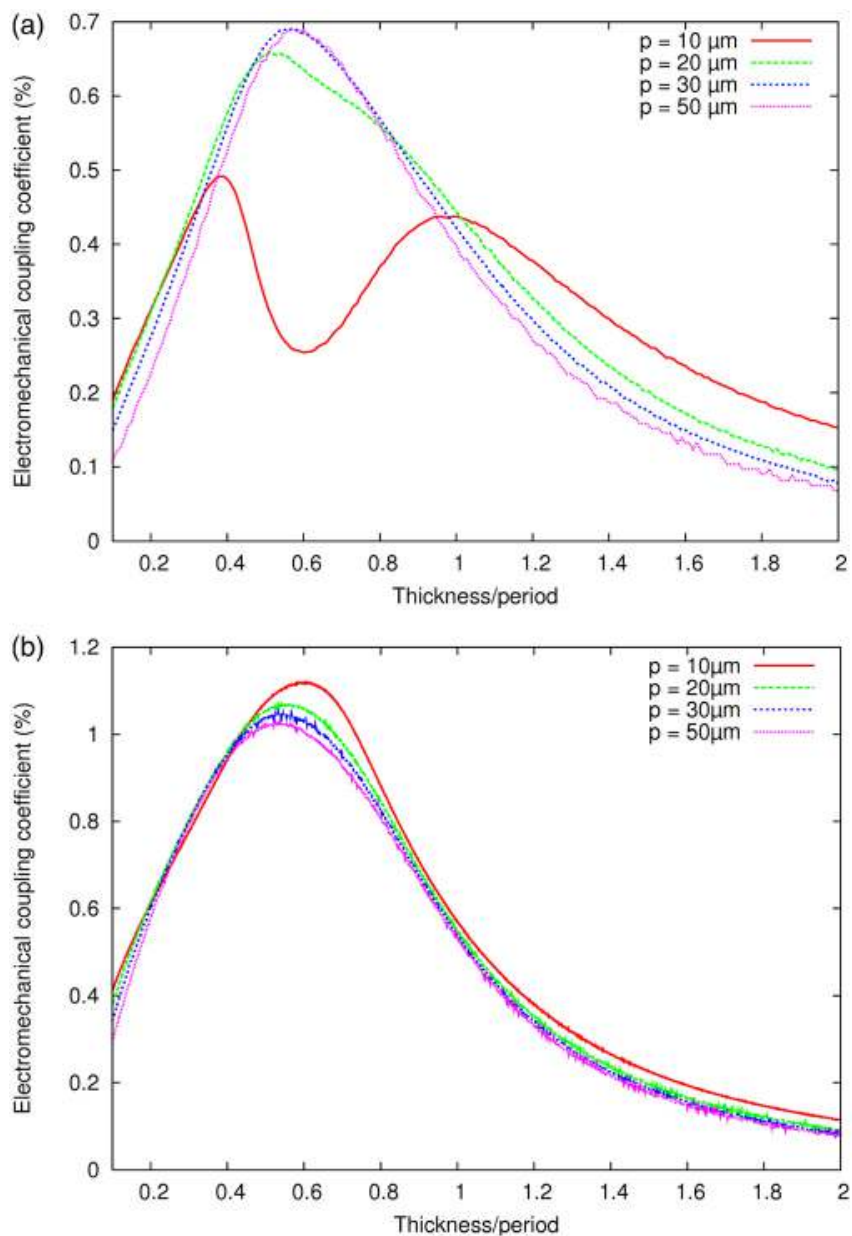

FIG. 9. Evolution of the isolated mode electromechanical coupling for small values of (e/p) ratio (ranging from 0.1 to 2 in our calculation) for several periods for $\mathrm{Si} / \mathrm{LiNbO}_{3} \mathrm{PPT} / \mathrm{Si}$ (a) and $\mathrm{Si} / \mathrm{LiTaO}_{3} \mathrm{PPT} / \mathrm{Si}$ (b) configurations. 

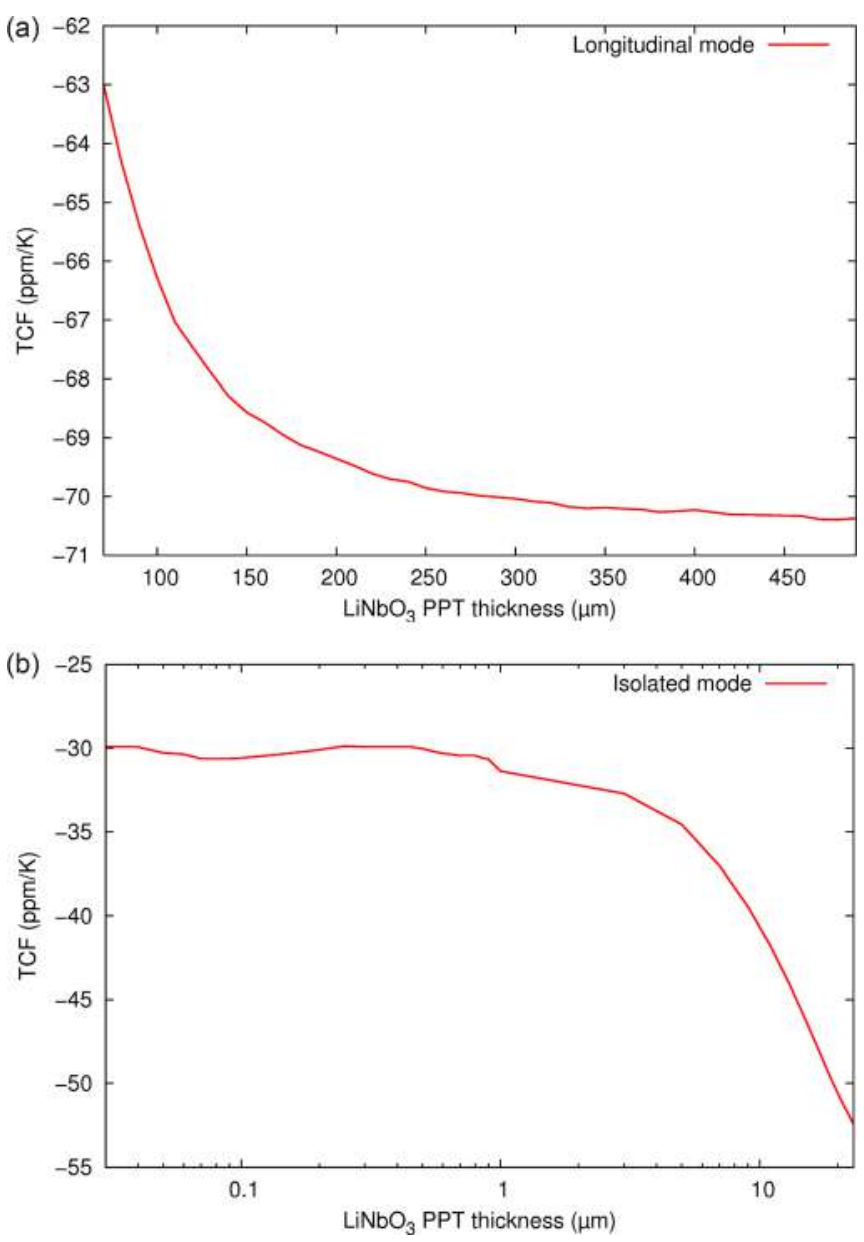

FIG. 10. TCF evolution for the longitudinal mode (a) and the one for the isolated mode (b) both for a $\mathrm{Si} / \mathrm{LiNbO}_{3} \mathrm{PPT}(\mathrm{p}=50 \mu \mathrm{m}) / \mathrm{Si}$ structure.

increases according to the PPT thickness. The smaller the PPT thickness, the smaller the TCF. Two TCF stabilizations are emphasized.

1. $\mathrm{TCF} \simeq-70 \mathrm{ppm} / \mathrm{K}$ for a PPT thickness higher than 400 $\mu \mathrm{m}$ (corresponding to the single $\mathrm{LiNbO}_{3}$ PPT TCF).

2. $\mathrm{TCF} \simeq-30 \mathrm{ppm} / \mathrm{K}$ for a PPT thickness tends to $0 \mu \mathrm{m}$ (corresponding to the silicon TCF). ${ }^{8}$

The TCF evolution of waves excited by a waveguide is summarized in Fig. 11.

Other configurations have been analyzed using different guiding materials such as silica or diamond. A few characteristics of different waveguide configurations are recalled in Table II.

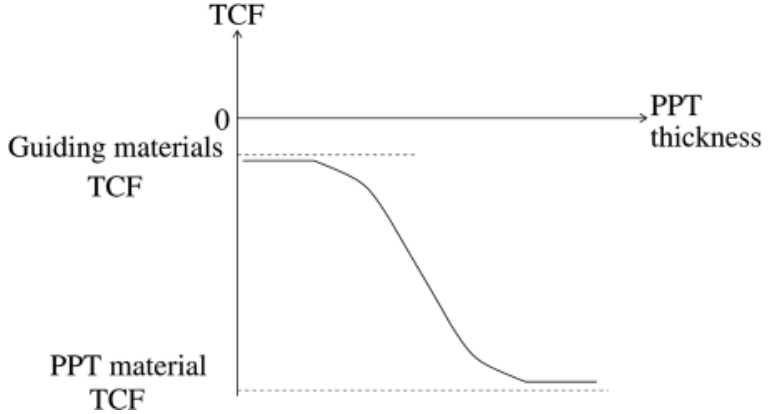

FIG. 11. TCF evolution of waves excited by a waveguide vs the PPT thickness.

A structure using a thinned PPT and silica on silicon allows the excitation of an elastic wave whose resonance frequency is insensitive to the temperature. Moreover, the use of diamond allows the guidance of elastic waves at higher phase velocity compatible with high frequency applications.

\section{CONCLUSION}

We have proposed a new acoustic resonator concept based on a periodically poled transducer implemented on a ferroelectric substrate $\left(\mathrm{LiNbO}_{3}\right.$ or $\left.\mathrm{LiTaO}_{3}\right)$ embedded between two silicon guiding substrates in order to create an acoustic waveguide. This structure is robust, integrable in the micro-electronic systems, and its temperature drift is optimized by the guiding materials choice (adding a silica layer).

PPT simulations point out the excitation of two main contributions corresponding to elliptical and longitudinal waves. The phase velocity of these waves is lower in a $\mathrm{LiTaO}_{3}$ PPT than in a $\mathrm{LiNbO}_{3}$ PPT. PPT-based waveguide simulations point out the excitation of elastic waves whose guidance depends on the PPT thickness. For a $500 \mu$ m-thick PPT, one main mode is excited with a phase velocity of $6500 \mathrm{~m} \mathrm{~s}^{-1}$ and a TCF of about $-70 \mathrm{ppm} / \mathrm{K}$ for a $\mathrm{LiNbO}_{3} \mathrm{PPT}$ and $5600 \mathrm{~m} \mathrm{~s}^{-1}$ and a TCF of about $-40 \mathrm{ppm} / \mathrm{K}$ for a $\mathrm{LiTaO}_{3}$ PPT. For a PPT whose thickness tends to $0 \mu \mathrm{m}$, only one mode is excited with a good spectral purity. A phase velocity of $5600 \mathrm{~m} \mathrm{~s}^{-1}$ and a TCF equal to about $-30 \mathrm{ppm} / \mathrm{K}$ are calculated for a $\mathrm{LiNbO}_{3}$ PPT and $4500 \mathrm{~m} \mathrm{~s}^{-1}$ with a TCF of about $-30 \mathrm{ppm} / \mathrm{K}$ for a $\mathrm{LiTaO}_{3} \mathrm{PPT}$. The TCF of this mode is limited by the guiding material. We have noted an optimal operating point for the $0.6(\mathrm{e} / \mathrm{p})$ ratio enabling to excite this wave with a maximum electromechanical coupling (about 1\%).

TABLE II. Theoretical summary of a few characteristics for different waveguide configurations.

\begin{tabular}{|c|c|c|c|c|}
\hline Structure & PPT material & Excited mode & Phase velocity $\left(\mathrm{m} \mathrm{s}^{-1}\right)$ & $\mathrm{TCF}(\mathrm{ppm} / \mathrm{K})$ \\
\hline \multirow[t]{2}{*}{ Substrate/PPT $(\mathrm{e}=500 \mu \mathrm{m}) /$ substrate } & $\mathrm{LiNbO}_{3}$ & Longitudinal & 6550 & $\simeq-70$ \\
\hline & $\mathrm{LiTaO}_{3}$ & Longitudinal & 5600 & $\simeq-40$ \\
\hline \multirow[t]{2}{*}{ Silicon/PPT $(\mathrm{e}=1 \mu \mathrm{m}) /$ Silicon } & $\mathrm{LiNbO}_{3}$ & Isolated & 5500 & $\simeq-30$ \\
\hline & $\mathrm{LiTaO}_{3}$ & Isolated & 5200 & $\simeq-30$ \\
\hline \multirow[t]{2}{*}{$\mathrm{Si} / \mathrm{SiO}_{2} / \mathrm{PPT}(\mathrm{e}=1 \mu \mathrm{m}) / \mathrm{SiO}_{2} / \mathrm{Si}$ for $5 \mu \mathrm{m}$ thick $\mathrm{SiO}_{2}$ layer } & $\mathrm{LiNbO}_{3}$ & Isolated & 5200 & $\simeq-0$ \\
\hline & $\mathrm{LiTaO}_{3}$ & Isolated & 4900 & $\simeq-0$ \\
\hline \multirow[t]{2}{*}{ Diamond/PPT $(\mathrm{e}=1 \mu \mathrm{m}) /$ Diamond } & $\mathrm{LiNbO}_{3}$ & Isolated & 8700 & $\simeq-10$ \\
\hline & $\mathrm{LiTaO}_{3}$ & Isolated & 7500 & $\simeq-10$ \\
\hline
\end{tabular}




\section{ACKNOWLEDGMENTS}

This work was supported by the French DGA (Direction Générale pour l'Armement) under Grant No. 07.34.020.

${ }^{1}$ E. Courjon, N. Courjal, W. Daniau, G. Lengaigne, L. Gauthier-Manuel, S. Ballandras, and J. Hauden, "Lamb wave transducers built on periodically poled Z-cut $\mathrm{LiNbO}_{3}$ wafers," J. Appl. Phys. 102, 114107 (2007).

${ }^{2}$ S. Ballandras, R. Petit, D. Gachon, and E. Courjon, Dispositifsàondes acoustiques d'interfaces, French patent 0805124 (2008).

${ }^{3}$ S. Ballandras, R. Lardat, M. Wilm, T. Pastureaud, A. Reinhardt, N. Champavert, W. Steichen, W. Daniau, V. Laude, R. Armati, and G. Martin, "A mixed finite element/boundary element approach to simulate complex guided elastic wave periodic transducers," J. Appl. Phys. 105, 014911 (2009).
${ }^{4}$ H. Kando, D. Yamamoto, M. Mimura, T. Oda, A. Shimizu, K. Shimoda, E. Takata, T. Fuyutsume, R. Kubo, M. Kadota, and Kyoto Murata Manufacturing Company Ltd., "6b-4 rf filter using boundary acoustic wave," Proc.IEEE Ultrason. Symp. 188-191 (2006).

${ }^{5}$ O. Elmazria, S. Zhgoon, L. Le Brizoual, F. Sarry, D. Tsimbal, and M. Abdou Djouadi, "Aln/ZnO/diamond structure combining isolated and surface acoustic waves," Appl. Phys. Lett. 95, 233503 (2009).

${ }^{6}$ A. K. Sarin Kumar, P. Paruch, J.-M. Triscone, W. Daniau, S. Ballandras, L. Pellegrino, D. Marré, and T. Tybell, "High-frequency surface acoustic wave device based on thin-film piezoelectric interdigital transducers," Appl. Phys. Lett. 85, 1757-1759 (2004).

${ }^{7}$ W. Steichen and S. Ballandras, "Composants acoustiques utilisés pour le filtrage," Techniques de l'ingénieur (E-2000) (2008).

${ }^{8}$ G. Kovacs, M. Anhorn, H. E. Engan, G. Visintini, and C. C. W. Ruppel, "Improved material constants for $\mathrm{LiNbO}_{3}$ and $\mathrm{LiTaO}_{3}$," Proc.-IEEE Ultrason. Symp. 1, 435-438 (1990). 